\section{Die neue Subjektivität}

Sozialpsychiatrie als wissenschaftliche Disziplin hat in den vergangenen Jahrzehnten einen schmerzhaften Entwicklungsprozess durchlaufen. Während wesentliche Reformimpulse in den 60er Jahren in Deutschland von sozialpsychiatrisch orientierten Universitätspsychiatern ausgingen, begann schon in den 70er Jahren eine Absetzbewegung aus der Universitätspsychiatrie in die Versorgungspsychiatrie, die nicht ohne schwerwiegende Folgen für die wissenschaftliche Entwicklung des Fachgebietes blieben.

Wenn auch mit unterschiedlicher Gewichtung war das Signal deutlich: Wo es Not gibt muss gehandelt werden und das Offensichtliche benötigt keine wissenschaftliche Reflexion. In den 70er und 80er Jahren wurden so Modellprogramme und Modellversuche zum Vehikel, neue Institutionstypen in die Regelversorgung einzuführen, wohingegen die obligatorische begleitende Versorgungsforschung zur Legitimationsforschung verkam. Eher selten erreichten Publikationen internationales Niveau. Sozialpsychiatrie war Haltung und gute Psychiatrie immer Sozialpsychiatrie.

Erkennbar schon in den 80er Jahren setzte eine Entwicklung ein, die bald die ganze psychiatrische Forschung ergriff, nämlich die Hinwendung zu dem was plakativ unter dem Begriff biologische Psychiatrie einzuordnen ist. Spätestens in den 90er Jahren wurde sozialpsychiatrische Forschung im deutschsprachigen Raum von der biologischen Forschung überrollt. Die Sozialpsychiatrie hatte dieser Entwicklung wenig entgegenzusetzen, weil es keine flächendeckende sozialpsychiatrische Forschung gab, die diesen Namen verdient hätte: Nicht wirklich erkenntnisorientiert, kaum internationale Forschungsstandards und keine Erfahrungen in der kompetitiven Drittmitteleinwerbung.

Matthias Angermeyer steht für den kleinen Kreis empirisch orientierter Sozialpsychiater, die den internationalen Wissenschaftsvergleich nicht zu scheuen brauchten. Er eröffnete in den 90er Jahren ein Forschungsfeld, das sich in verschiedenen Abwandlungen bald großer Beliebtheit erfreuen sollte, nämlich die Meinungsumfrage. Bekannt wurden seine repräsentativen Umfragen im Hinblick auf die Einstellung der Bevölke-

Psychiat Prax 2001; 28: $53-54$

(c) Georg Thieme Verlag Stuttgart · New York

ISSN 0303-4259
Wulf Rössler

Psychiatrische Universitätsklinik Zürich

rung zu psychisch Kranken und im Besonderen, welche negativen Auswirkungen die Attentate auf Lafontaine und Schäuble auf die öffentliche Meinung zu psychisch Kranken hatten. Ihm war es damit gelungen, solide wissenschaftliche Standards mit einem klassischen sozialpsychiatrischen Anliegen, nämlich die Stellung psychisch Kranker in ihrer sozialen Umwelt zu verbinden.

Um der „Meinungsumfrage“ zum heutigen Erfolg zu verhelfen, brauchte es allerdings noch ein paar Zutaten. Zum einen wurden damit einer Dimension psychiatrischen Handelns wieder Gehör verschafft, die in der Flut objektiver Befunde der biologischen Psychiatrie unterzugehen drohte, nämlich dem subjektiven Erleben von Krankheit und Krankheitsbewältigung. Tatsächlich konnte die Sozialpsychiatrie mit diesem Ansatz im wissenschaftlichen Diskurs, Terrain zurückerobern. Und vor allen Dingen hatte die Sozialpsychiatrie einen wichtigen Verbündeten, nämlich die Betroffenen selbst, deren Anliegen damit auch zu den Fachleuten transportiert werden konnten. Die subjektiven Anliegen wurden durch diesen Forschungsansatz in den Rang objektiver Tatsachen erhoben. Dies blieb nicht ohne Rückwirkung auf andere psychiatrische Teilgebiete. Themenbereiche wie subjektive Lebensqualität, Belastung Angehöriger, Stigma etc. sind nicht mehr der alleinigen sozialpsychiatrischen Diskussion vorbehalten, sondern haben inzwischen breit Eingang gefunden in alle Teilbereiche der Psychiatrie.

Zum anderen aber eröffnete sich den Fachleuten in der Sozialpsychiatrie mit der Meinungsumfrage ein neues Wissenschaftsfeld, das scheinbar ohne die sonst übliche Mühsal sozialpsychiatrischer Forschung vergleichsweise schnell Publikationen versprach - Publikationen, die in dem zunehmend härter werdenden Konkurrenzgeschäft wissenschaftlicher Karrieren immer notwendiger wurden. Dieser Forschungsansatz hatte für die wissenschaftsorientierten Sozialpsychiater darüber hinaus den unschätzbaren Vorteil, nicht im Verdacht zu stehen, vorrangig dem eigenen Interesse einer Wissenschaftskarriere zu dienen.

Ganz nebenbei hat damit auch noch das kritisch-rationale Forschungsparadigma Einzug in die sozialpsychiatrische Forschung gehalten. Dieses Paradigma setzt rigorose Standards in der Überprüfung von Fragestellungen und Hypothesen, während hingegen der Entstehungszusammenhang von Fragestellungen weniger Aufmerksamkeit findet. Diese Entwicklung 
blieb nicht ganz ohne Auswirkung auf sozialpsychiatrische Fachgesellschaften. So hat z.B. die Deutsche Gesellschaft für soziale Psychiatrie einen eigenen Wissenschaftspreis ausgeschrieben, wobei der Vorstand sich die letzte Entscheidung über den Preisträger vorbehalten hat, der von dem mit Wissenschaftlern besetzten Preiskomitee benannt wurde. $\mathrm{Zu}$ Recht fürchtet sich die Fachgesellschaft davor, die Definitionsmacht darüber zu verlieren, was „gute“ und „schlechte“ Fragestellungen und „richtige“ und „falsche“ Antworten darauf sind.

Neben all diesen positiven Entwicklungen, die sich aus der Hinwendung zum Forschungsgegenstand „Subjektivität“ ergeben, treten allmählich und immer deutlicher auch einige Nachteile in den Vordergrund. Zum einen ist eine zunehmende Trivialität publizierter Ergebnisse unverkennbar. Der Erkenntniswert übersteigt häufig nicht unser Alltagswissen. Zum anderen, so wichtig persönliche Krankheitserfahrung und -verarbeitung ist, unser Erkenntnisinteresse reicht darüber hinaus. Die Dominanz der Subjektivität verdrängt nicht selten den Anspruch sozialpsychiatrischer Forschung, nicht nur das Vordergründige zu beschreiben, sondern dahinter stehende (soziale) Zusammenhänge zu verstehen und zu erklären.

Die internationale sozialpsychiatrische Forschung hat in den letzten Jahrzehnten einen breiten Wissensbestand über den Einfluss der physischen und sozialen Umwelt auf Entstehung und Verlauf psychischer Störungen erarbeitet. Wenn die Variablen der physischen und sozialen Umwelt scheinbar weniger starken Einfluss auf Erkrankungsrisiko und -verlauf nehmen als biologische Variablen, heißt das nicht, dass sie weniger bedeutsam wären als vielmehr, dass entweder

- die Variabilität der sozialen Umwelt wenig systematisiert und damit weniger analysierbar ist, oder

- die Variabilität der sozialen Umwelt in den forschungsbeherrschenden Ländern nicht mehr sehr groß ist, um wesentlich zur Varianzaufklärung beizutragen.

Im Hinblick auf die erste Hypothese schlägt der bekannte amerikanische Soziologe W. Harrison deshalb vor, in Analogie zum Genomprojekt, das sich die Katalogisierung der menschlichen Gene zum Ziel gesetzt hat, ein vergleichbares Projekt zu beginnen, mit dem Ziel die menschliche Umwelt systematisch zu erfassen. Im Hinblick auf die zweite Hypothese sollten wir unser Augenmerk verstärkt auf Entwicklungs- und Schwellenländer richten, wo der Einfluss der physischen und sozialen Umwelt z. B. durch Urbanisierung oder Migration evident ist. Dieses aufklärerische Potenzial der Sozialpsychiatrie droht aber verloren zu gehen, wenn wir uns allzu bequem der „neuen Subjektivität" hingeben.

Prof. Dr. med. Dipl.-Psych. W. Rössler

Psychiatrische Universitätsklinik

Militärstr. 8

8021 Zürich

Schweiz

\section{BUCHBESPRECHUNG}

\section{Veränderungen bei der Therapeutin}

Die Psychoanalyse als Vorreiterin der Psychotherapie hat sich schwer getan, über die Veränderungen nachzudenken, die die therapeutische Arbeit in der Person der Behandelnden auslöst. Dies steht in gewissem Widerspruch zum Plädoyer für die Beziehung als entscheidendem Wirkfaktor der Therapie. Der Begriff der Gegenübertragung zeigt, dass Nachdenken stattgefunden hat, aber in der ursprünglichen Annahme, dass Gefühle, Reaktionen oder Veränderungen allein auf das Konto der Kranken gehe. Die Entwicklung der Gegenübertragungskonzepte zeichnet die Geschichte dieser Reflexionen nach. Sie nähert sich einem interaktiven Modell des Veränderungsprozesses. Soeben ist aus dem Bereich der Hypnose ein Band mit Reflexionen über „Veränderungen im Therapeuten“ erschienen. 16 Autorinnen und Autoren berichten über subjektive Entwicklungen und Veränderungen im Gefolge der Begegnungen mit Patientinnen und Patienten. Es dürfte kein Zufall sein, dass einschneidende Lebensereignisse der Anlass fast aller Therapien waren: Die Diagnose von Karzinomen; infauste Prognosen; Tod naher Angehöriger; Foltererfahrung. Im Wesentlichen handelt es sich um lebendige Fallgeschichten in bester psychotherapeutischer Tradition. Sie werden aus der Perspektive der Rückwirkungen auf die Therapeutin dargestellt. Einzelne Beiträge enthalten lohnende Anregungen. Die systematische Untersuchung des Veränderungsprozesses steht aber noch aus.

Ulrike Hoffmann-Richter, Luzern

Kahn S, Fromm E (eds). Changes in the Therapist. 2001. 222 S. (Lawrence Erlbaum Associates, London.) 